\title{
Pooling-analysis for diagnostic and prognostic value of MiRNA-100 in various cancers
}

\author{
Zhe Dou ${ }^{1, *}$, Shuai Lin ${ }^{1, *}$, Cong Dai ${ }^{1, *}$, Ye Lu ${ }^{2}$, Tian Tian ${ }^{1}$, Meng Wang $^{1}$, Xinghan Liu ${ }^{1}$, \\ Yi Zheng ${ }^{1}$, Peng Xu ${ }^{1}$, Shanli Li ${ }^{1}$, Qianwen Sheng ${ }^{1}$, Yujiao Deng ${ }^{1}$ and Zhijun Dai ${ }^{1}$ \\ ${ }^{1}$ Department of Oncology, Second Affiliated Hospital of Xi'an Jiaotong University, Xi'an 710004, China \\ ${ }^{2}$ Department of Student Affairs, Second Affiliated Hospital of Xi'an Jiaotong University, Xi'an 710004, China \\ ${ }^{*} \mathrm{ZD}, \mathrm{SL}$ and $\mathrm{CD}$ have contributed equally to this work \\ Correspondence to: Zhijun Dai, email: dzj0911@126.com, dzj0911@ xjtu.edu.cn \\ Keywords: miRNA-100, diagnosis, prognosis, meta-analysis \\ Received: December 14, $2016 \quad$ Accepted: May 15, $2017 \quad$ Published: June 27, 2017 \\ Copyright: Dou et al. This is an open-access article distributed under the terms of the Creative Commons Attribution License 3.0 \\ (CC BY 3.0), which permits unrestricted use, distribution, and reproduction in any medium, provided the original author and source \\ are credited.
}

\section{ABSTRACT}

Many studies manifested miRNA-100 was deregulated in various cancers, which indicated that miRNA-100 might be a potential biomarker of cancer diagnosis and prognosis. However, the role of miRNA-100 was still uncertain. We searched for qualified studies using PubMed, EMBASE, Web of Science, Cochrane library and CNKI databases. The diagnostic effect was evaluated by the pooled sensitivity, specificity, and other indexes. Pooled hazard ratios (HRs) with $\mathbf{9 5 \%}$ confidence intervals (CIs) for overall survival (OS) were calculated to assess the prognostic value. This meta-analysis included 7 and 19 studies about diagnosis and prognosis, respectively. The results of pooled sensitivity, specificity, positive likelihood ratio (PLR), negative likelihood ratio (NLR) and diagnostic odds ratio (DOR) were 0.75 (95\%CI: $0.71-0.78), 0.74$ (95\%CI: 0.69-0.78), 2.61 (95\%CI: 1.81-3.76), 0.33 (95\%CI: 0.24-0.45), 8.46 (95\%CI: $4.85-$ 14.77), respectively. And, the area under SROC curve (AUC) was 0.8141 . We also found that lower expression of miRNA-100 in cancer tissues could significantly predict poorer prognosis in overall cancer ( $\mathrm{HR}=0.59,95 \% \mathrm{CI}: 0.39-0.90)$, especially in genital system tumors $(\mathrm{HR}=0.42,95 \% \mathrm{CI}: 0.27-0.66, \mathrm{P}=0.431)$, bladder cancer $(\mathrm{HR}=0.21,95 \% \mathrm{CI}$ : $0.06-0.73, P=0.143)$ and esophageal squamous cell carcinoma $(\mathrm{HR}=0.26,95 \% \mathrm{CI}$ : $0.13-0.52, P=0.164)$. Our studies concluded that miRNA-100 has a certain value in diagnosis and it may indicate a poor prognosis of cancers.

\section{INTRODUCTION}

Cancer is always a fearsome disease because of its high mortality. It was estimated by GLOBOCAN that there were about 14.1 million new cancer cases and 8.2 million deaths occurring in 2012 and about 57\% of cases and $65 \%$ of cancer deaths in developed countries worldwide [1]. Therefore, cancer has become a compelling health problem, and early diagnosis is particularly important in the treatment of cancer, but it is difficult because of the limitations in present diagnostic methods. Imaging examination and biopsy have the disadvantages of their invasive and harmful procedure, and many current biomarkers lack high accuracy in clinical diagnosis. In addition, it's difficult to predict the clinical outcomes of cancer, which significantly varied in different people. At present, the research of biomarkers has made rapid development [2]. It's highly needed to seek for new biomarkers that can exert on detection or diagnosis in early-age or estimate the prognosis of patients.

With a length of 19-25 nucleotides, microRNAs (miRNAs) are small non-coding RNAs which could regulate gene expression by blinding 3' untranslated region (3'UTR) of their target mRNA and inhibiting gene translation. These miRNAs are considered as 
gene regulators at post transcriptional gene level [3]. As a member of miRNA-99a family, miRNA-100 is located on chromosome11 at 11q24.1 (Gene ID: 406892) and has been demonstrated to play a potential role in cell proliferation, tumorigenesis, angiogenesis and differentiation $[4,5]$. Dysregulated expression of miRNA-100 is correlated with cancer diagnosis and prognosis [6]. Many studies suggested miRNA-100 as an oncogene or a tumor suppress gene. However, their conclusions remain controversial. Recent studies demonstrated obviously down-regulated expression of miRNA-100 in many tumor tissues, such as bladder cancer $[7,8]$, lung cancer $[9,10]$, esophageal squamous cell carcinoma [11, 12], epithelial ovarian cancer [13, 14], and other cancers $[15-19,20]$, indicating that it may have a relationship with poorer prognosis in cancer patients. But, evidence from some other studies showed opposite results in several types of cancer [21-25]. In addition, the diagnostic accuracy and the prognostic significance of miRNA-100 remain unclear. With due consideration of the limitations of a single study, we performed this systematic review and meta-analysis to evaluate the diagnostic and prognostic value of miRNA-100 in various cancers.

\section{RESULTS}

\section{Literature search}

A total of 175 studies from a primary literature were searched in PubMed, EMBASE, Web of Science, Cochrane library and CNKI. After reviewing titles and abstracts manually, some studies were excluded due to their irrelevance to the analysis, or because they were review articles, duplicate studies, letters, animal experiments or laboratory studies. Then, we reviewed full texts and omitted 26 studies that were unrelated to diagnosis or prognosis and 10 studies without sufficient data to obtain the crucial data for analysis. Finally, 24 available articles were included. Among those articles, one article researched the diagnostic as well as the prognostic value of miRNA-100, meanwhile, it was divided into two studies because of its different investigations in plasma and tissue for diagnosis [16]. One study for prognosis was omitted because of its investigation in serum alone [26]. Finally, we enrolled 19 eligible prognostic studies and 7 eligible diagnostic studies from 6 articles in this metaanalysis (Figure 1).

\section{Diagnostic meta-analysis}

\section{Study characteristics}

7 eligible articles of cancer diagnosis were published from 2010 to 2016, involving a total of 883 participants. These participants were from China, Egypt, Poland, and Mexico. Various types of tumors contain bladder cancer, gastric cancer, endometrioid endometrial carcinoma, esophageal squamous cell carcinoma, acute lymphoblastic leukemia and prostate cancer. Specimens contain serum/ plasma, tissue, and urine. And, all studies adopted the approach of quantitative reverse transcription polymerase chain reaction (qRT-PCR) to measure the expression of miRNA-100. The main characteristics of these eligible studies were listed in Table 1 [16, 27-31]. The quality of the studies according to QUADAS-2 tool was good, which was summarized in Figure 2.

\section{Diagnostic accuracy and threshold analysis}

Firstly, we used the receiver operating characteristic curve (ROC) to identify whether it exist threshold effect. The result showed that there was no heterogeneity from threshold effect. What's more, the Spearman's correlation coefficient in this meta-analysis was 0.393 $(\mathrm{P}=0.383)$, which confirmed the result was objective. According to results of the inconsistency index $\left(\mathrm{I}^{2}\right)$, we chose the random-effect model to calculate all indexes. The results of the pooled sensitivity, specificity, positive likelihood ratio (PLR), negative likelihood ratio (NLR), diagnostic odds ratio (DOR) were 0.75 (95\%CI: 0.71$0.78), 0.74$ (95\%CI: 0.69-0.78), 2.61 (95\%CI: 1.81-3.76), 0.33 (95\%CI: $0.24-0.45), 8.46$ (95\%CI: 4.85-14.77), respectively (Figure 3). Moreover, as shown in Figure 4, the area under curve (AUC) was 0.8141 , suggesting that miRNA-100 had a certain value in diagnosis.

\section{Prognostic meta-analysis}

\section{Study characteristics}

A total of 19 studies with 2009 patients were included in this prognostic meta-analysis. Among those, patients in 16 studies were from China [7-12, 14, 15, 17-21, 23-25] and the other 3 studies were from Germany [22], Poland [16], and Iran [13]. All the studies were published from 2012 to 2016. The tumors types involved to colorectal cancer $(n=2)$, lung cancer $(\mathrm{n}=3)$, bladder cancer $(\mathrm{n}=2)$, esophageal squamous cell carcinoma $(\mathrm{n}=2)$, acute leukemia $(\mathrm{n}=2)$, breast cancer $(\mathrm{n}=1)$, pancreatic ductaladeno carcinoma $(\mathrm{n}=1)$, hepatocellular carcinoma $(n=1)$, renal cell carcinoma $(n=1)$, endometrioid endometrial carcinoma $(\mathrm{n}=1)$, small cell carcinoma of the cervix $(n=1)$, and epithelial ovarian cancer $(n=2)$. The numbers of patients ranged from 44 to 204 . The expression level of miRNA-100 was measured by qRT-PCR, and HRs and $95 \%$ CIs for OS was extracted from each studies. There were 10 studies based on univariate analysis and 9 studies with multivariate analysis. The main characteristics of the eligible studies were listed in Table 2, which also included the scores according to the Newcastle-Ottawa scale (NOS).

\section{Meta-analysis and subgroup analysis}

Obvious heterogeneity was found among these 19 studies for the correlation between the expression of miRNA-100 and overall survival (OS) $\left(\mathrm{I}^{2}=85.2 \%\right)$, so we used the random-effect model to combine hazard 
ratio (HR) value and $95 \% \mathrm{CI}$. With a pooled $\mathrm{HR}$ for OS of 0.59 (95\%CI: 0.39-0.90), our findings demonstrated that decreased expression of miRNA-100 in tissue predicted a poor clinical outcome (Figure 5). Likewise, the subgroup analysis was integrated into the investigation of heterogeneous sources and the relationship between HRs value and other variables, including sample size, types of cancers, methods, and countries (Table 3). Apparently, we found a significant relationship with lower HR in genital system tumors $(\mathrm{HR}=0.42,95 \% \mathrm{CI}$ : $0.27-0.66 \mathrm{P}=0.431)$, bladder cancer $(\mathrm{HR}=0.21,95 \% \mathrm{CI}: 0.06-0.73, \mathrm{P}=0.143)$ and esophageal squamous cell carcinoma $(\mathrm{HR}=0.26$, 95\%CI: 0.13-0.52, $\mathrm{P}=0.164)$. Moreover, the results showed there was obvious statistical significance among Chinese subjects ( $\mathrm{HR}=0.55,95 \% \mathrm{CI}$ : 0.35-0.86) and for studies with larger sample sizes $(>100$ subjects) $(\mathrm{HR}=0.44,95 \% \mathrm{CI}$ : $0.30-0.64)$. Considering the difference between analysis methods, we conducted subgroup analysis by analysis

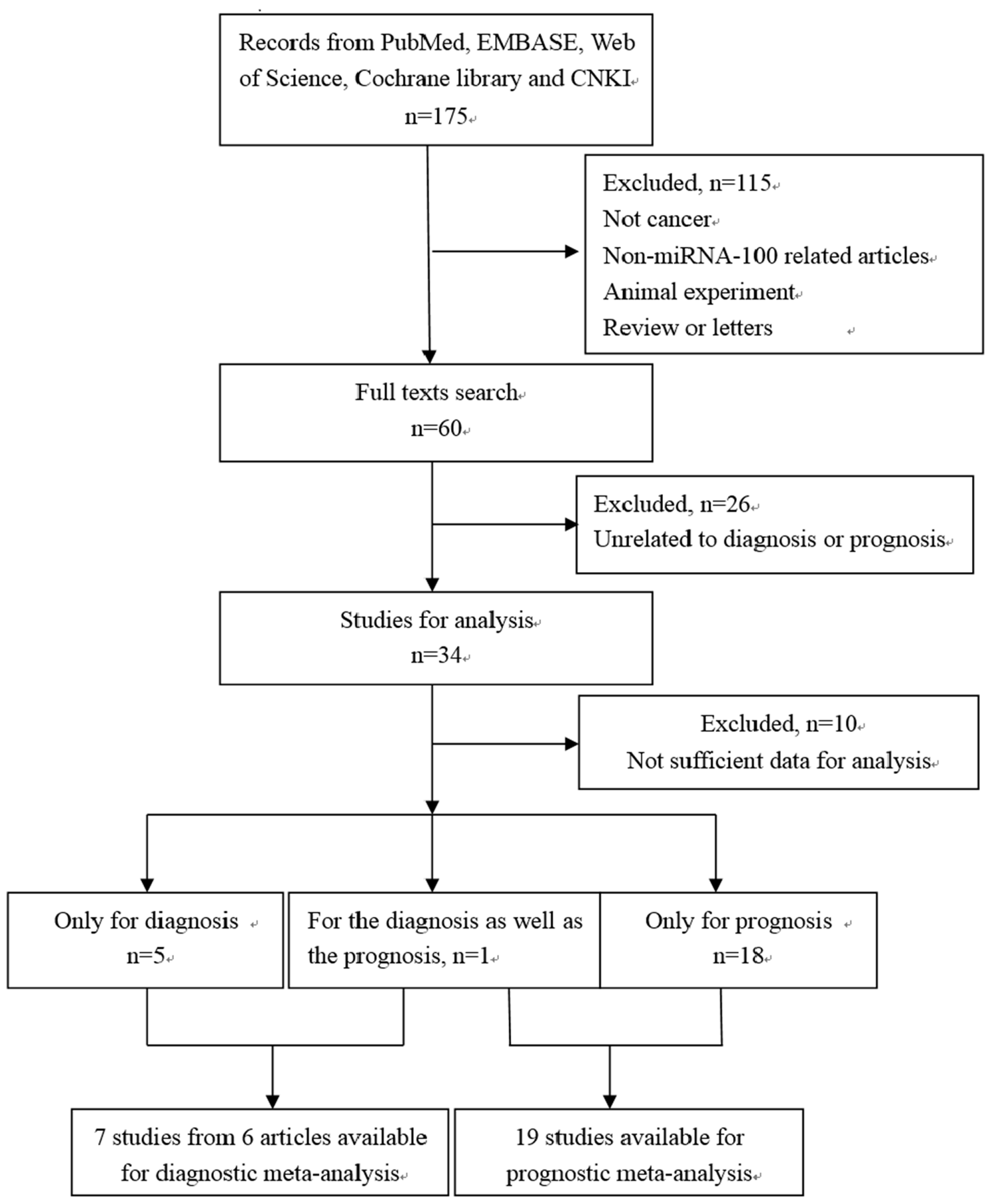

Figure 1: The flow diagram of the study selection process. 
Table 1: Main characteristics of eligible studies in diagnostic systematic review

\begin{tabular}{|c|c|c|c|c|c|c|c|c|c|c|c|c|}
\hline Author & Year & Country & Tumor type & Patients & Controls & Specimen & Method & $\mathbf{A U C}$ & TP & FP & FN & $\mathbf{T N}$ \\
\hline Tarek et al & 2016 & Egypt & $\mathrm{BC}$ & 70 & 62 & serum & qRT-PCR & $0.823(0.728-0.917)$ & 63 & 21 & 7 & 41 \\
\hline Wang et al & 2014 & China & $\mathrm{GC}$ & 50 & 47 & serum & qRT-PCR & $0.71(0.61-0.82)$ & 36 & 20 & 15 & 27 \\
\hline \multirow[t]{2}{*}{ Anna et al } & 2012 & Poland & EEC & 34 & 14 & plasma & qRT-PCR & $0.740(0.592-0.857)$ & 22 & 3 & 12 & 11 \\
\hline & & & & 73 & 31 & tissue & qRT-PCR & $0.652(0.548-0.746)$ & 63 & 16 & 10 & 16 \\
\hline Zhang et al & 2010 & China & ESCC & 149 & 100 & serum & qRT-PCR & $0.817(0.763-0.870)$ & 95 & 19 & 54 & 81 \\
\hline Menha et al & 2016 & Egypt & ALL & 85 & 25 & $\begin{array}{l}\text { serum/ } \\
\text { plasma }\end{array}$ & qRT-PCR & $0.87(0.779-0.934)$ & 70 & 0 & 15 & 25 \\
\hline Alberto et al & 2016 & Mexico & prostate cancer & 73 & 70 & urine & qRT-PCR & $0.738(0.652-0.823)$ & 51 & 13 & 22 & 57 \\
\hline
\end{tabular}

$\mathrm{BC}=$ bladder cancer, $\mathrm{GC}=$ gastric cancer, $\mathrm{EEC}=$ endometrioid endometrial carcinoma, ESCC = esophageal squamous cell carcinoma, $\mathrm{ALL}=$ acute lymphoblastic leukemia, qRT-PCR = quantitative reverse transcription polymerase chain reaction, $\mathrm{AUC}=$ the area under the $\mathrm{SROC}$ curve, TP $=$ truepositive, $\mathrm{FP}=$ false-positive, $\mathrm{FN}=$ false-negative, $\mathrm{TN}=$ true negative.

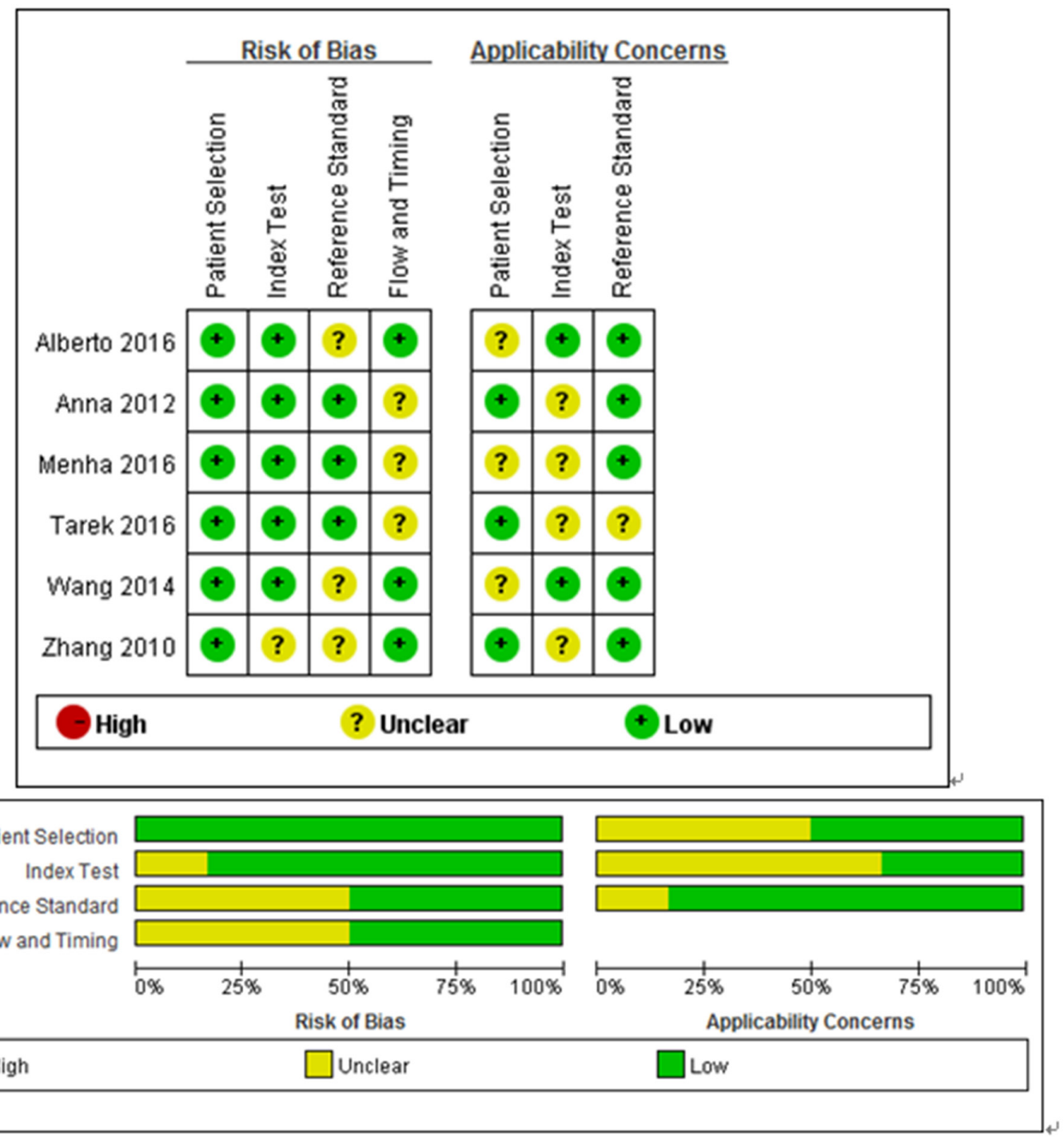

Figure 2: Details of quality assessment by the QUADAS-2 tool. “-” in red and "+" in green mean high risk and low risk respectively. "?" in yellow means unclear risk. 
methods, showing that the results were meaningful among the studies used univariate analyses $(\mathrm{HR}=0.56,95 \% \mathrm{CI}$ : 0.38-0.82, shown in Table 3).

\section{Meta aggression and sensitivity analysis}

We conducted the meta-regression based on publication year, country, sample size, analysis method, tumor type and follow-up period, with an intention of exploring the potential source of heterogeneity in our analysis. However, there was no obvious evident revealed from the results that either of the above covariates in this meta-regression contributed to heterogeneity (shown in Table 4).

Meanwhile, we performed sensitivity analysis on the pooled HR for OS about the expression of miRNA-100 in patients. The selected studies were sequentially removed to investigate whether any single study could have an influence on the pooled HRs. As displayed in Figure 6, the results were stable and not significantly affected by each individual study.

\section{Publication bias}

Begg's and Egger's tests were used to evaluate the publication bias of the included studies (Figure 7). Begg's funnel plot did not reveal any evidence of significant asymmetry. With the P value of Egger's test of being 0.800 , it indicated no significant existence of publication bias.

\section{DISCUSSION}

Many investigators reported miRNA-100 in various cancers as a novel molecular target. And, mammalian target of rapamycin (mTOR) gene and insulin-like growth factor 1 receptor (IGF1R) are direct target of miRNA-100 in bladder cancer, acute myelocytic leukemia, endometrioid endometrial carcinoma and so

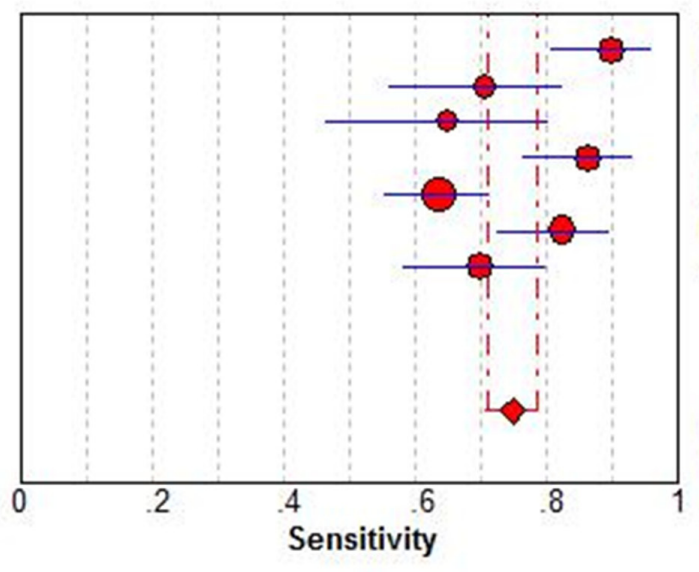

Tarek et Wang et Anna et Anna et Zhang et Menha et Alberto et

Pooled Sensitivity $=0.75$ (0.71 to 0.78$)$

Chi-square $=30.95 ; \mathrm{df}=6(\mathrm{p}=0.0000)$

Inconsistency (l-square) $=80.6 \%$

\section{a: Forest plot of estimated sensitivity}

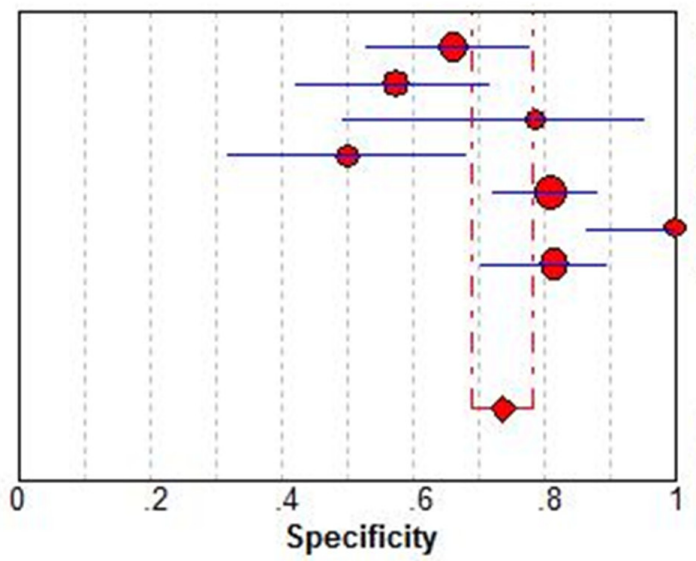

Tarek et Wang et Anna et Anna et Zhang et Menha et Alberto et

Pooled Specificity $=0.74$ (0.69 to 0.78$)$

Chi-square $=36.38 ; d f=6(p=0.0000)$

Inconsistency (l-square) $=83.5 \%$
Specificity $(95 \% \mathrm{CI})$

$0.66 \quad(0.53-0.78)$

$0.57 \quad(0.42-0.72)$

$0.79 \quad(0.49-0.95)$

$0.50 \quad(0.32-0.68)$

$0.81 \quad(0.72-0.88)$

$1.00 \quad(0.86-1.00)$

$0.81 \quad(0.70-0.90)$

b: Forest plot of estimated specificity

Figure 3: Forest plots of estimated sensitivity (a) and specificity (b) for miRNA-100 in the diagnostic analysis. 
on $[12,16,20,32]$. MiRNA-100 could suppress the related proteins of the $\mathrm{IGF} / \mathrm{mTOR}$ signaling cascade in different cancers. Overexpression of miR-100 inhibited the expressions of IGF1R and mTOR by targeting its 3 'UTR at posttranscriptional gene level, so that interfer cell proliferation and survival signaling in some types of tumors. And, miRNA-100 can also exert as a tumor suppressor in many cancers by targeting polo-like kinase 1 (PLK1) [9, 14, 19]. MiRNA-100 was found to significantly inhibit the expression of PLK1 and other proteins, which has a vital effect on cell growth, apoptosis, development and drug resistance. In addition, several studies reported that miR-100 regulated apoptosis in gastric tumor cells and breast cancer cells [33, 34], and they declared miR-100 antagonism triggers apoptosis by inhibiting ubiquitination-mediated p53 degradation [35]. Besides, Cyr61 and RBSP3 was discovered a potential target of miRNA-100 for regulation. Hence, these miRNA-100 related cellular and molecular pathways may provide some ideas for new therapeutic targets in many types of cancers.
The present meta-analysis for diagnosis showed us a pooled sensitivity of 0.75 , a pooled specificity of 0.74 , DOR of 8.46, AUC of 0.1841 , and a PLR of 2.61, which illustrated that there was an approximately 3-fold higher possibility of being miRNA-100 positive for patients with cancer in comparison to those without. And, a NLR of 0.33 mean the probability of miRNA-100 negative patients having cancers was $33 \%$, which suggested that the diagnosis of miRNA-100 existed a certain degree of accuracy but not high enough. But it still had a great advantage compared to other traditional serum-based biomarkers, such as the sensitivities for lung cancer of CEA, Cyfra21-1, SCC and NSE were 46.2\%, 40.0\%, $43.1 \%$, and $46.2 \%$, respectively. The clinical significance of single biomarker was not ideal. So it may achieve a better diagnostic accuracy through uniting other biomarkers or clinical examinations.

For prognostic value, some studies indicated the low miR-100 expression in bladder cancer predicts unfavorable prognosis and it might regulate tumor metastasis or other related processes about tumorigenesis by inhabiting

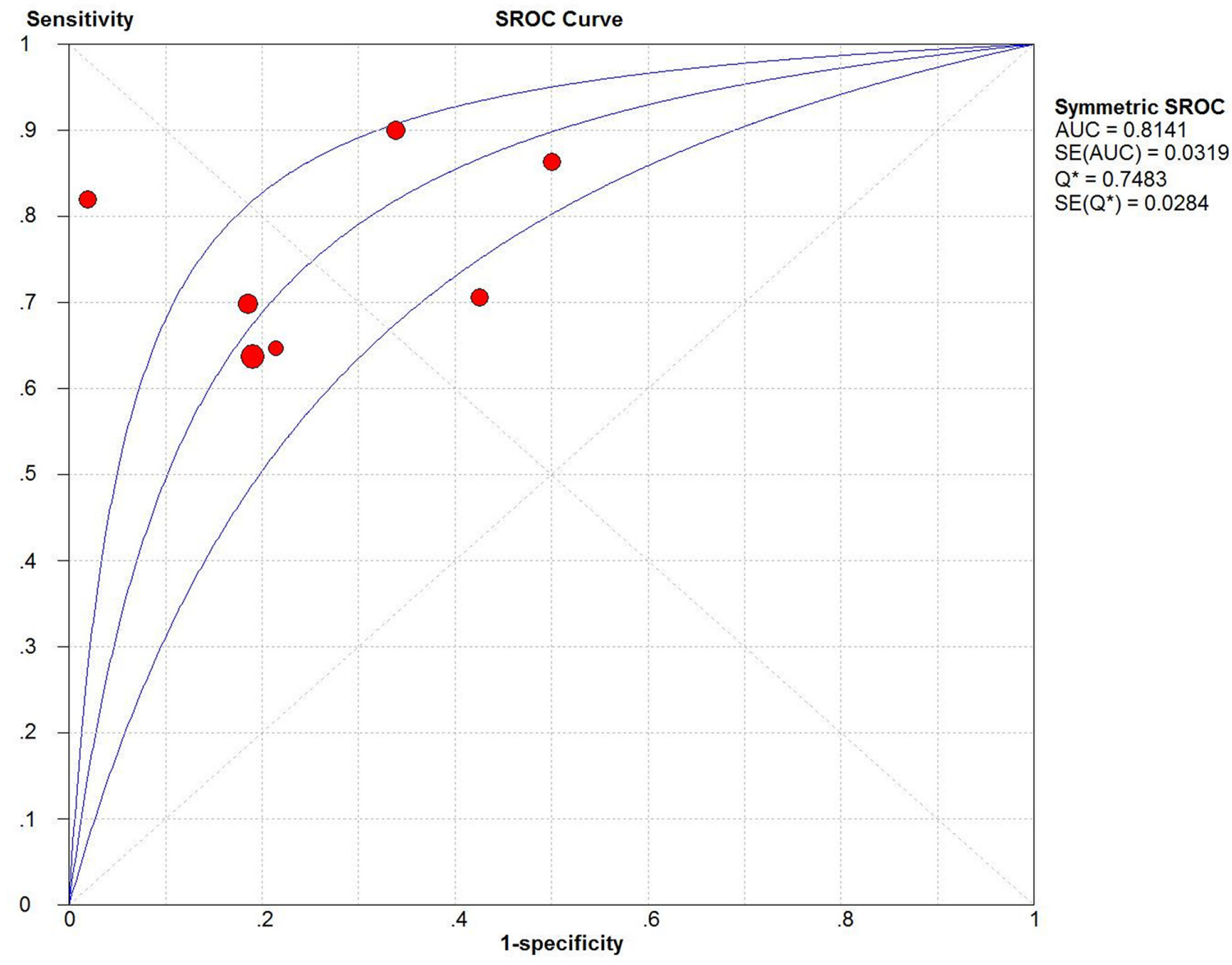

Figure 4: Summary receiver operating characteristic (SROC) Curves of miRNA-100. 
Table 2: Main characteristics of eligible studies in prognostic systematic review

\begin{tabular}{|c|c|c|c|c|c|c|c|c|c|c|c|}
\hline Author & Year & Country & $\begin{array}{c}\text { Tumor } \\
\text { type }\end{array}$ & $\begin{array}{c}\text { Sample } \\
\text { size }\end{array}$ & Specimen & Method & Cutoff & Outcomes & Follow-up (months) & $\begin{array}{l}\text { Survival } \\
\text { analysis }\end{array}$ & NOS \\
\hline Susan et al & 2016 & Iran & EOC & 55 & tissue & qRT-PCR & - & OS & $40(7-90)$ & $\mathrm{U}$ & 8 \\
\hline Zhang et al & 2015 & China & breast cancer & 204 & tissue & TCGA database & - & OS & $10-170$ & $\mathrm{U}$ & 8 \\
\hline Zhang et al & 2015 & China & $\mathrm{CRC}$ & 172 & tissue & qRT-PCR & median & OS & 41 & $\mathrm{U}$ & 7 \\
\hline Sameer et al & 2015 & Germany & PDAC & 98 & tissue & qRT-PCR & 5 & OS & $0-120$ & $\mathrm{U}, \mathrm{M}$ & 8 \\
\hline Luo et al & 2015 & China & NSCLC & 48 & tissue & qRT-PCR & median & OS & 18 & $\mathrm{U}$ & 7 \\
\hline Cao et al & 2015 & China & $\mathrm{BC}$ & 92 & tissue & qRT-PCR & - & OS & $0-50$ & $\mathrm{U}, \mathrm{M}$ & 7 \\
\hline Zhou et al & 2014 & China & ESCC & 120 & tissue & qRT-PCR & median (1.77) & OS & $22.62(2.63-76.87)$ & $\mathrm{U}, \mathrm{M}$ & 8 \\
\hline Chen et al & 2014 & China & $\mathrm{CRC}$ & 138 & tissue & qRT-PCR & median (1.26) & OS & $5-60$ & U.M & 8 \\
\hline Li et al & 2013 & China & ALL & 111 & bone marrow & qRT-PCR & - & OS & $0-60$ & $\mathrm{U}$ & 8 \\
\hline Chen et al & 2013 & China & $\mathrm{HCC}$ & 134 & tissue & qRT-PCR & - & OS & $0-60$ & U,M & 7 \\
\hline Wang et al & 2013 & China & $\mathrm{RCC}$ & 96 & tissue & qRT-PCR & median (5.5) & OS & $81.8(25.2-133.6)$ & $\mathrm{U}, \mathrm{M}$ & 8 \\
\hline Sun et al & 2013 & China & ESCC & 61 & tissue & qRT-PCR & - & OS & $0-100$ & $\mathrm{U}$ & 7 \\
\hline Wang et al & 2012 & China & NSCLC & 92 & tissue & qRT-PCR & median $(0.02)$ & OS & $6(1-33)$ & $\mathrm{U}, \mathrm{M}$ & 7 \\
\hline Anna et al & 2012 & Poland & EEC & 104 & tissue & qRT-PCR & - & OS & $10-150$ & $\mathrm{U}$ & 7 \\
\hline Wang et al & 2012 & China & $\mathrm{BC}$ & 126 & tissue & qRT-PCR & - & OS & 36 & $\mathrm{U}, \mathrm{M}$ & 7 \\
\hline Huang et al & 2012 & China & SCCC & 44 & tissue & qRT-PCR & 6.515 & OS & $23.6(2-70)$ & $\mathrm{U}, \mathrm{M}$ & 7 \\
\hline Peng et al & 2012 & China & $\mathrm{EOC}$ & 98 & tissue & qRT-PCR & median $(0.14)$ & OS & $0-60$ & $\mathrm{U}$ & 8 \\
\hline Liu et al & 2012 & China & NSCLC & 110 & tissue & qRT-PCR & - & OS & $0-65$ & $\mathrm{U}$ & 7 \\
\hline Bai et al & 2012 & China & AML & 106 & bone marrow & qRT-PCR & median (10.8) & OS & $35(10-86)$ & $\mathrm{U}$ & 8 \\
\hline
\end{tabular}

$\mathrm{M}=$ multivariate, $\mathrm{U}=$ univariate, $\mathrm{qRT}-\mathrm{PCR}=$ quantitative reverse transcription polymerase chain reaction, $\mathrm{CRC}=$ colorectal cancer, $\mathrm{PDAC}=$ pancreatic ductal adenocarcinoma, $\mathrm{NSCLC}=$ non small cell lung cancer, $\mathrm{BC}=$ bladder cancer, $\mathrm{EEC}=$ endometrioid endometrial carcinoma, $\mathrm{ESCC}=$ esophageal squamous cell carcinoma, $\mathrm{AML}=$ acute myelocytic leukemia, $\mathrm{ALL}=$ acute lymphoblastic leukemia, $\mathrm{HCC}=$ hepatocellular carcinoma, $\mathrm{RCC}=$ renal cell carcinoma, $\mathrm{GC}=\mathrm{gastric}$ cancer, $\mathrm{SCCC}=$ small cell carcinoma of the cervix, EOC $=$ epithelial ovarian cancer, $\mathrm{OS}=$ overall survival, NOS $=$ Newcastle-Ottawa scale.

mTOR [36-41]. A study in EEC observed that decreased miRNA-100 in EEC tissues and up-regulated miRNA-100 in plasma by targeting mTOR making it as a promising biomarker for diagnosis and prognosis [16]. Some studies on EOC, SCCC and NSCLC found miR-100 was significantly decreased in cancer tissues in comparison to healthy people, which appeared that low miR-100 was a poor prognostic biomarker by targeting PLK1 in patients $[9,11,13,14,19,42]$ and some researches in HCC, CRC and ESCC hold the similar view [11, 12, 17, 43-45]. But, there are also some studies had different opinions in some types of cancer. Some studies showed miRNA-100 was up-regulated in cancer tissues of AML, RCC, PDAC and NSCLC, causing the result was quite opposite [21-23, 46]. Then, we conducted this meta-analysis for prognosis, and we found lower miRNA-100 expression may predict a poorer outcome in various cancers, and the predictive efficacy was more significant in genital system tumors, $\mathrm{BC}$ and ESCC. HRs were significant for studies in Chinese subjects, larger sample sizes ( $>100$ subjects) and by univariate analyses. So we think miRNA-100 may be a potential biomarker for prognostic. However, as the results of sensitivity analysis and meta-regression, we couldn't find the resource of heterogeneity, so we summarized the data using the random-effect model. Considering the source of heterogeneity, we speculated that the heterogeneity may be caused by the cut-off value of miRNA-100 expression, which was not been reported explicit values in many articles, and multiple factors may influence the heterogeneity together, such as the difference of selection criteria for patients in various tumor types and studies, the diversified clinicopathological characteristics of patients in different studies, the specific method of randomization and blind, the random errors in studies and so on.

Recently, a meta-analysis was reported about prognostic value of miRNA-100 in cancers. They suggested that patients with lower expression of miRNA-100 in cancer tissue had poorer survival in a variety of carcinomas, which was similar to our result in prognostic meta-analysis [47]. There have many differences between us, such as published time, sample size, statistical software, data processing and so on. Firstly, six studies published before October 2013 were included in that study, while we extracted data from 19 available studies published before October 2016 for prognostic and 7 studies for diagnostic meta-analysis. Secondly, in 
the measures of data processing, they only calculated the pooled HR for OS and investigated publication bias in their analysis, without exploring the source of heterogeneity in that study. However, we carried out the subgroup analysis by some variables and discovered that there were significant results especially in genital system tumors, bladder cancer and esophageal squamous cell carcinoma. Meanwhile, with an aim of probing deeply into the source of heterogeneity, we executed the meta-regression, subgroup analysis and sensitivity analysis. What's more, the diagnostic meta-analysis was also referred to the investigation of the diagnostic and prognostic significance ofmiRNA-100 in various cancers. So, we have more advantages in comparison with previous study. Our study is the first meta-analysis to research the diagnostic and prognostic value of miRNA-100 in various cancers. In addition, our study may be more comprehensive and abundant due to our efforts in subgroup analysis, metaregression and so on. Besides, many new studies have been incorporated into our article, which contributed to a more reliable conclusion.

Nevertheless, this study still exists some limitations. Firstly, research and sample size in single tumor type was relatively small, which probably influenced the research in single cancer. Secondly, our studies have a very high ratio of data in Chinese patients, which may limits its application to global range. Thirdly, some HRs could not be extracted from primary studies and needed to calculate from the Kaplan-

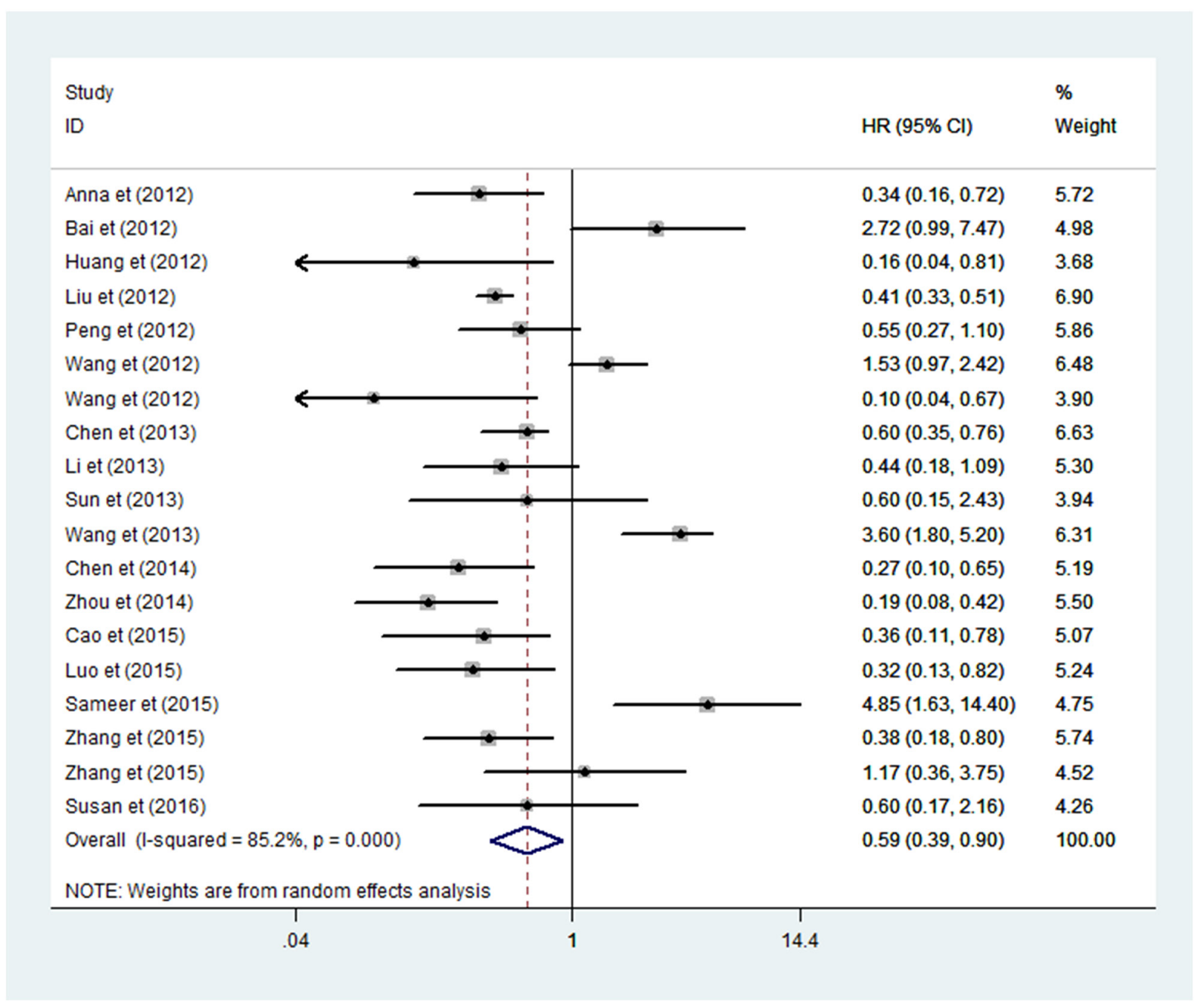

Figure 5: Forrest plots of studies evaluating HRs of high miRNA-100 expression as compared to low expression for cancer. $\mathrm{CI}=$ confidence interval, $\mathrm{HR}=$ hazard ratio. 
Table 3: Main results of the pooled analysis

\begin{tabular}{|c|c|c|c|c|c|c|c|}
\hline \multirow[t]{2}{*}{ Survival } & \multirow[t]{2}{*}{ Variables } & \multirow[t]{2}{*}{ No. of studies } & \multicolumn{3}{|c|}{$\begin{array}{c}\text { Rondom-effects model or fixed- } \\
\text { effects model }\end{array}$} & \multicolumn{2}{|c|}{ Heterogeneity } \\
\hline & & & $\begin{array}{c}\text { No. of } \\
\text { patients }\end{array}$ & $\begin{array}{l}\text { Pooled } \\
\text { HR }\end{array}$ & $95 \% \mathrm{CI}$ & $I^{2}$ & $\mathbf{P}$ \\
\hline \multirow[t]{16}{*}{ OS } & All & 19 & 2009 & 0.59 & $0.39-0.90$ & $85.20 \%$ & 0.000 \\
\hline & Type & & & & & & \\
\hline & genital system tumors & 4 & 301 & 0.42 & $0.27-0.66$ & $0.00 \%$ & 0.431 \\
\hline & digestive system & 6 & 723 & 0.65 & $0.29-1.47$ & $80.40 \%$ & 0.000 \\
\hline & respiratory system & 3 & 250 & 0.60 & $0.23-1.62$ & $92.60 \%$ & 0.000 \\
\hline & urinary system & 3 & 314 & 0.54 & $0.04-4.74$ & $93.90 \%$ & 0.000 \\
\hline & others & 3 & 421 & 0.74 & $0.23-2.37$ & $80.9 \%$ & 0.005 \\
\hline & Sample & & & & & & \\
\hline & $>100$ & 10 & 1325 & 0.44 & $0.30-0.64$ & $67.70 \%$ & 0.001 \\
\hline & $<100$ & 9 & 684 & 0.83 & $0.41-1.70$ & $84.40 \%$ & 0.000 \\
\hline & Country & & & & & & \\
\hline & China & 16 & 1752 & 0.55 & $0.35-0.86$ & $85.80 \%$ & 0.000 \\
\hline & Other countries & 3 & 257 & 0.98 & $0.19-5.10$ & $87.20 \%$ & 0.000 \\
\hline & Method & & & & & & \\
\hline & Univariate & 10 & 1069 & 0.56 & $0.38-0.82$ & $52.40 \%$ & 0.032 \\
\hline & Multivariate & 9 & 940 & 0.57 & $0.28-1.17$ & $89.80 \%$ & 0.000 \\
\hline
\end{tabular}

Table 4: Meta-regression analyses of potential source of heterogeneity

\begin{tabular}{lcccccc}
\hline Heterogeneity factors & Coefficient & SE & $\mathbf{Z}$ & $\mathbf{p}$ & \multicolumn{2}{c}{$\mathbf{9 5 \%}$ CI } \\
\cline { 5 - 7 } & & & & & $\mathbf{L L}$ & $\mathbf{U L}$ \\
\hline Publication year & 0.029 & 0.181 & 0.16 & 0.875 & -0.354 & 0.412 \\
Country & 0.565 & 0.661 & 0.85 & 0.404 & -0.829 & 1.960 \\
Number of patients & -0.001 & 0.006 & -0.16 & 0.876 & -0.144 & 0.012 \\
Analysis method & 0.400 & 0.472 & 0.85 & 0.408 & -0.595 & 1.396 \\
Tumor types & -0.106 & 0.140 & -0.76 & 0.459 & -0.402 & 0.189 \\
Follow-up & 0.006 & 0.006 & 1.07 & 0.299 & $-0,006$ & 0.018 \\
\hline
\end{tabular}

$\mathrm{SE}=$ standard error, $\mathrm{CI}=$ confidence interval, $\mathrm{LL}=$ lower limit, $\mathrm{UL}=$ upper limit.

Meier survival curves using indirectly method, which may cause a certain calculation error. Finally, it exists an obvious heterogeneity in our meta-analysis, according to the sensitivity analysis and meta-regression, and we could just supposed the resource of heterogeneity. Therefore, further studies in the future are expected to draw a more definitive conclusion.

In summary, we concluded miRNA-100 had a certain value in diagnosis despites its diagnostic accuracy 
was not high enough, and it had a significant value as a prognostic biomarker. Therefore, investigating the expression of miRNA-100 in various cancers may provide a new thinking into cancer prevention and therapeutic strategy, and the different expression level of miRNA-100 in cancers may indicate different endings of patients.

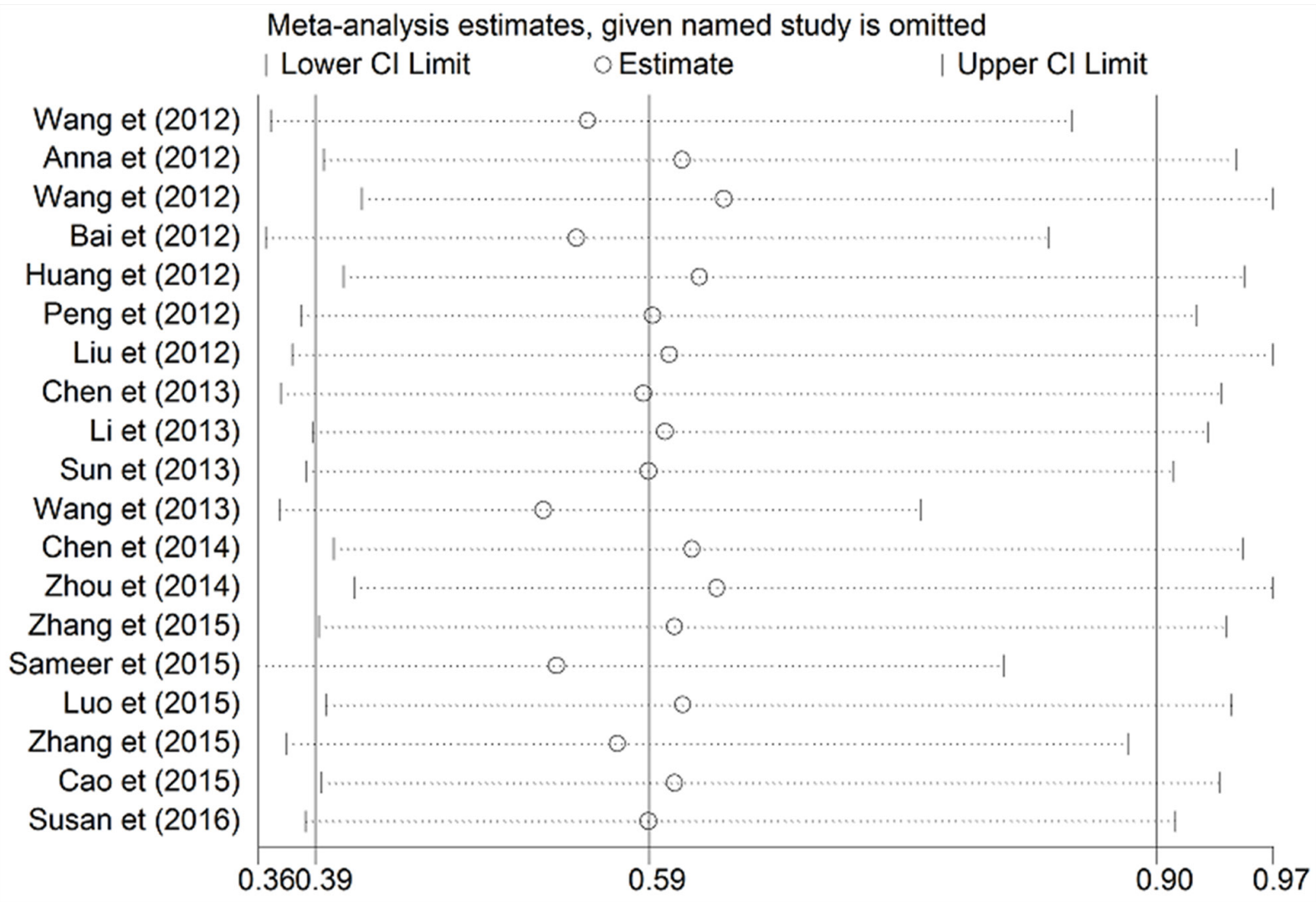

Figure 6: Sensitivity analysis on the pooled hazard ratio for miRNA-100 and overall survival of patients.
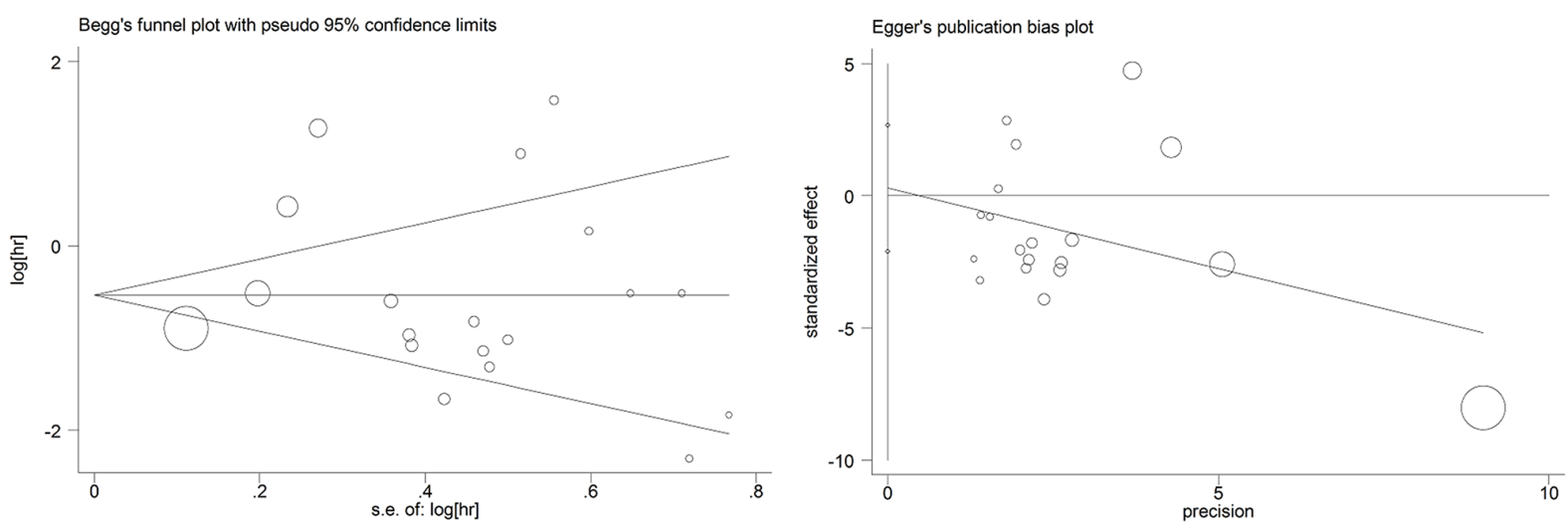

Figure 7: Begg's and egger's funnel plots for all of the included studies reported with overall survival. 


\section{METHODS}

\section{Search strategy and selection criteria}

This meta-analysis was conducted following the guidelines of the Meta-analysis of Observational Studies in Epidemiology and Preferred Reporting Items for Systematic Reviews and Meta-Analyses groups [48]. We carefully searched for the relevant articles in PubMed, Web of Science, EMBASE and CNKI (up to October 31,2016 ) assessing the diagnostic accuracy and the prognostic significance of miRNA-100 in various types of cancers. The keywords such as microRNA-100/ miRNA-100/miR-100, cancer/carcinomas, prognosis and diagnosis were used. Moreover, the reference articles from all associated articles were also found and scanned manually to retrieve any additional eligible studies.

The eligible studies must fit the following inclusion criteria: (i) the study investigates the diagnostic or prognostic value of miRNA-100 in patients with various carcinomas; (ii) for diagnosis, they must provide enough information that we could obtain the crucial data directly or through calculation, such as true-positive (TP), false-positive (FP), falsenegative (FN), and true negative (TN); (iii) for prognosis, they must provide enough information so that we could extract directly or indirectly HRs with 95\% CIs for OS; (iv) for prognosis, they measured the expression of miRNA-100 in tumor tissues.

Articles will be excluded by following criteria: (i) duplicate studies; (ii) review articles or letters; (iii) non-original articles; (iv) animal experiments and laboratory studies. Two reviewers independently searched and identified all articles, resolving disagreements by consensus in research group.

\section{Data extraction}

Two investigators independently made judgments and extraction of the relevant data, settling disagreements through consensus adjudication by research group. The extracted data included name of the first author, publication year, number of patients, cancer types, specimen, test method, diagnostic results (AUC, TP, FP, TN, FN) and related data for prognostic (cut-off, follow-up, HR, 95\% CI). If not obtaining diagnostic results directly, we calculate the data using their sensitivity and specificity. We collect HRs and their $95 \%$ CIs preferentially from multivariate or univariate analyses in the original article, and $\mathrm{HR}>1$ means higher expression of miRNA-100 in tumor tissues that may have a poorer prognosis in cancer patients. If not available, we calculate HRs with corresponding 95\% CIs from Kaplan-Meier curves through Engauge 4.0 software. Meanwhile, study quality of studies in diagnostic metaanalysis was rated by Quality Assessment of Diagnostic
Accuracy Studies (QUADAS-2) assessment tool. We also use the Newcastle-Ottawa (NOS) scale to evaluate the quality of each included study, which score ranges from " 0 " to " 9 " and a score $\geq 6$ indicates high quality.

\section{Statistical analysis}

As for diagnostic meta-analysis, we calculated and combined sensitivity, specificity, PLR, NLR, DOR, and corresponding 95\% 95\% CIs based on the key data (TP, FP, FN, TN), ROC and Spearman correlation coefficient were applied to verify a threshold effect. We measured the heterogeneity by the $\mathrm{I}^{2} . \mathrm{I}^{2}>50 \%$ indicated that significant heterogeneity exists in studies, then we implemented the random-effect model to calculate the related indexes (DerSimonian-Laird method), otherwise, the fixed-effect model was selected (MantelHaenszel method). Simultaneously, the diagnostic accuracy was assessed by the area under the SROC curve (AUC) from summary receiver operative curve (SROC). In addition, in the case of two-sided $\mathrm{p}$ values across the board, $\mathrm{P}<0.05$ was considered statistical significant. The diagnostic meta-analysis were performed with Meta- Disc software, version 1.4 (Unit of Clinical Biostatistics, Ramony 94 Cajal Hospital, Madrid, Spain) [49].

For prognostic meta-analysis, HRs and $95 \% \mathrm{CIs}$ of OS were weighted and pooled to estimate the contact between expression of miRNA-100 and prognostic significance in various cancer patients. Higgins I-squared statistic was used to measure statistical heterogeneity, we adopted random-effect model for severe heterogeneity with $\mathrm{I}^{2}>50 \%$, while fixed-effect model for the absence of heterogeneity with $\mathrm{I}^{2}<50 \%$ [50]. The confounder contribution to heterogeneity was explored through the approach of subgroup analysis, meta-regression and sensitivity analysis. Besides, we adopted Begg's and Egger's test to study whether it exists publication bias. All the results were considered statistical significant at twosided P-value of 0.05 . The prognostic meta-analysis was conducted with STATA statistical software, version 14.0 (Stata Corporation, College Station, TX, USA).

\section{CONFLICTS OF INTEREST}

The authors declare no conflicts of interest.

\section{REFERENCES}

1. Torre LA, Bray F, Siegel RL, Ferlay J, Lortet-Tieulent J, Jemal A. Global cancer statistics, 2012. CA Cancer J Clin. 2015; 65: 87-108. doi: 10.3322/caac.21262.

2. Ferracin M, Veronese A, Negrini M. Micromarkers: miRNAs in cancer diagnosis and prognosis. Expert Rev Mol Diagn. 2010; 10: 297-308. doi: 10.1586/erm.10.11. 
3. Yang W, Lee DY, Ben-David Y. The roles of microRNAs in tumorigenesis and angiogenesis. Int J Physiol Pathophysiol Pharmacol. 2011; 3: 140-55.

4. Jansson MD, Lund AH. MicroRNA and cancer. Mol Oncol. 2012; 6: 590-610. doi: 10.1016/j.molonc.2012.09.006.

5. Berardi E, Pues M, Thorrez L, Sampaolesi M. miRNAs in ESC differentiation. Am J Physiol Heart Circ Physiol. 2012; 303: H931-9. doi: 10.1152/ajpheart.00338.2012.

6. Iorio MV, Croce CM. MicroRNA dysregulation in cancer: diagnostics, monitoring and therapeutics. A comprehensive review. EMBO Mol Med. 2012; 4: 143-59. doi: 10.1002/ emmm.201100209.

7. Cao YH, Zhang HH, Xu HF, Duan YJ, Li Q, Huang B. Prognostic role of microRNA-100 in patients with bladder cancer. Genet Mol Res. 2015; 14: 15948-54. doi: 10.4238/2015.December.7.6.

8. Wang S, Xue S, Dai YQ, Yang JF, Chen ZJ, Fang XW, Zhou WS, Wu W, Li QW. Reduced expression of microRNA-100 confers unfavorable prognosis in patients with bladder cancer. Diagn Pathology. 2012; 7: 159. doi: Artn 15910.1186/1746-1596-7-159.

9. Liu J, Lu KH, Liu ZL, Sun M, De W, Wang ZX. MicroRNA-100 is a potential molecular marker of nonsmall cell lung cancer and functions as a tumor suppressor by targeting polo-like kinase 1. BMC Cancer. 2012; 12: 519. doi: Artn 51910.1186/1471-2407-12-519.

10. Luo J, Chen B, Ji XX, Zhou SW, Zheng D. Overexpression of miR-100 inhibits cancer growth, migration, and chemosensitivity in human NSCLC cells through fibroblast growth factor receptor 3. Tumour Biol. $2015 \mathrm{Aug} 28$. [Epub ahead of print]. doi: 10.1007/s13277-015-3850-Z.

11. Sun J, Chen Z, Tan X, Zhou F, Tan F, Gao Y, Sun N, Xu X, Shao K, He J. MicroRNA-99a/100 promotes apoptosis by targeting mTOR in human esophageal squamous cell carcinoma. Med Oncol. 2013; 30: 411. doi: 10.1007/ s12032-012-0411-9.

12. Zhou S, Yang B, Zhao Y, Xu S, Zhang H, Li Z. Prognostic value of microRNA-100 in esophageal squamous cell carcinoma. J Surg Res. 2014; 192: 515-20. doi: 10.1016/j. jss.2014.07.005.

13. Azizmohammadi S, Azizmohammadi S, Safari A, Kosari N, Kaghazian M, Yahaghi E, Seifoleslami M. The role and expression of miR-100 and miR-203 profile as prognostic markers in epithelial ovarian cancer. Am J Transl Res. 2016; 8: 2403-10.

14. Peng DX, Luo M, Qiu LW, He YL, Wang XF. Prognostic implications of microRNA-100 and its functional roles in human epithelial ovarian cancer. Oncol Rep. 2012; 27: 1238-44. doi: 10.3892/or.2012.1625.

15. Zhang B, Zhao R, He Y, Fu X, Fu L, Zhu Z, Fu L, Dong JT. MicroRNA 100 sensitizes luminal A breast cancer cells to paclitaxel treatment in part by targeting mTOR. Oncotarget. 2015; 7: 5702-14. doi: 10.18632/oncotarget.6790.
16. Torres A, Torres K, Pesci A, Ceccaroni M, Paszkowski T, Cassandrini P, Zamboni G, Maciejewski R. Deregulation of miR-100, miR-99a and miR-199b in tissues and plasma coexists with increased expression of mTOR kinase in endometrioid endometrial carcinoma. BMC Cancer. 2012; 12: 369 .

17. Chen $\mathrm{P}, \mathrm{Xi}$ Q, Wang Q, Wei P. Downregulation of microRNA-100 correlates with tumor progression and poor prognosis in colorectal cancer. Med Oncol. 2014; 31: 235. doi: 10.1007/s12032-014-0235-X.

18. Chen P, Zhao X, Ma L. Downregulation of microRNA-100 correlates with tumor progression and poor prognosis in hepatocellular carcinoma. Mol Cell Biochem. 2013; 383: 49-58. doi: 10.1007/s11010-013-1753-0.

19. Huang L, Lin JX, Yu YH, Zhang MY, Wang HY, Zheng M. Downregulation of six microRNAs is associated with advanced stage, lymph node metastasis and poor prognosis in small cell carcinoma of the cervix. PLoS One. 2012; 7: e33762. doi: 10.1371/journal.pone.0033762.

20. Li XJ, Luo XQ, Han BW, Duan FT, Wei PP, Chen YQ. MicroRNA-100/99a, deregulated in acute lymphoblastic leukaemia, suppress proliferation and promote apoptosis by regulating the FKBP51 and IGF1R/mTOR signalling pathways. Br J Cancer. 2013; 109: 2189-98. doi: 10.1038/ bjc.2013.562.

21. Wang G, Chen L, Meng J, Chen M, Zhuang L, Zhang L. Overexpression of microRNA-100 predicts an unfavorable prognosis in renal cell carcinoma. Int Urol Nephrol. 2013; 45: 373-9. doi: 10.1007/s11255-012-0374-y.

22. Dhayat SA, Abdeen B, Kohler G, Senninger N, Haier J, Mardin WA. MicroRNA-100 and microRNA-21 as markers of survival and chemotherapy response in pancreatic ductal adenocarcinoma UICC stage II. Clin Epigenetics. 2015; 7: 132. doi: 10.1186/s13148-015-0166-1.

23. Bai J, Guo A, Hong Z, Kuai W. Upregulation of microRNA-100 predicts poor prognosis in patients with pediatric acute myeloid leukemia. Onco Targets Ther. 2012; 5: 213-9. doi: 10.2147/OTT.S36017.

24. Wang T, Lv M, Shen S, Zhou S, Wang P, Chen Y, Liu B, Yu L, Hou Y. Cell-free microRNA expression profiles in malignant effusion associated with patient survival in nonsmall cell lung cancer. PLoS One. 2012; 7: e43268. doi: 10.1371/journal.pone.0043268.

25. Zhang S, Yuan W, Tang W, Xu C, Ma J. Expression of microRNA-100 and its relation with prognosis of colorectal cancer. [Article in Chinese]. Chin J Oncol. 2015; 37: 603-8.

26. Adusumilli PS, Wu C, Wang C, Guan X, Liu Y, Li D, Zhou X, Zhang Y, Chen X, Wang J, Zen K, Zhang CY, Zhang C. Diagnostic and prognostic implications of a serum miRNA panel in oesophageal squamous cell carcinoma. PLoS One. 2014; 9: e92292. doi: 10.1371/journal.pone.0092292.

27. Motawi TK, Rizk SM, Ibrahim TM, Ibrahim IA. Circulating microRNAs, miR-92a, miR-100 and miR-143, as non-invasive biomarkers for bladder cancer diagnosis. 
Cell Biochem Funct. 2016; 34: 142-8. doi: 10.1002/ cbf.3171.

28. Salido-Guadarrama AI, Morales-Montor JG, RangelEscareno C, Langley E, Peralta-Zaragoza O, Cruz Colin JL, Rodriguez-Dorantes M. Urinary microRNA-based signature improves accuracy of detection of clinically relevant prostate cancer within the prostate-specific antigen grey zone. Mol Med Rep. 2016; 13: 4549-60. doi: 10.3892/ mmr.2016.5095.

29. Swellam M, El-Khazragy N. Clinical impact of circulating microRNAs as blood-based marker in childhood acute lymphoblastic leukemia. Tumour Biol. 2016; 37: 10571-6. doi: 10.1007/s13277-016-4948-7.

30. Wang H, Wang L, Wu Z, Sun R, Jin H, Ma J, Liu L, Ling R, Yi J, Wang L, Bian J, Chen J, Li N, et al. Three dysregulated microRNAs in serum as novel biomarkers for gastric cancer screening. Med Oncol. 2014; 31: 298. doi: 10.1007/ s12032-014-0298-8.

31. Zhang C, Wang C, Chen X, Yang C, Li K, Wang J, Dai J, Hu Z, Zhou X, Chen L, Zhang Y, Li Y, Qiu H, et al. Expression profile of microRNAs in serum: a fingerprint for esophageal squamous cell carcinoma. Clin Chem. 2010; 56: 1871-9. doi: 10.1373/clinchem.2010.147553.

32. Liu MX, Li J, Geng YL, Wang YC, Li J, Chen YJ, Ali G, Tarver SL, Wen YF, Sun WJ. Correlation study of knowledge and behavior regarding breast care among female undergraduate students in China. Asian Pac J Cancer Prev. 2014; 15: 10943-7.

33. Yang G, Gong Y, Wang Q, Wang Y, Zhang X. The role of miR-100-mediated Notch pathway in apoptosis of gastric tumor cells. Cell Signal. 2015; 27: 1087-101. doi: 10.1016/j. cellsig.2015.02.013.

34. Gong Y, He T, Yang L, Yang G, Chen Y, Zhang X. The role of miR-100 in regulating apoptosis of breast cancer cells. Sci Rep. 2015; 5: 11650. doi: 10.1038/srep11650.

35. Yang G, Gong Y, Wang Q, Wang L, Zhang X. miR-100 antagonism triggers apoptosis by inhibiting ubiquitinationmediated p53 degradation. Oncogene. 2017; 36: 1023-37. doi: 10.1038/onc.2016.270.

36. Xu C, Zeng Q, Xu W, Jiao L, Chen Y, Zhang Z, Wu C, Jin T, Pan A, Wei R, Yang B, Sun Y. miRNA-100 inhibits human bladder urothelial carcinogenesis by directly targeting mTOR. Mol Cancer Ther. 2013; 12: 207-19. doi: 10.1158/1535-7163.MCT-12-0273.

37. Ratert NM, Jung M, Lioudmer P, Mollenkopf HJ, Wagner I. MiRNA profiling identifies candidate miRNAs for bladder cancer diagnosis and clinical outcome. J Mol Diagn. 2013; 15: 695-705.

38. Oliveira JC, Brassesco MS, Morales AG, Pezuk JA, Fedatto PF, da Silva GN, Scrideli CA, Tone LG. MicroRNA-100 acts as a tumor suppressor in human bladder carcinoma 5637 cells. Asian Pac J Cancer Prev. 2011; 12: 3001-4.

39. Dip N, Reis ST, Timoszczuk LS, Viana NI, Piantino CB, Morais DR, Moura CM, Abe DK, Silva IA, Srougi M,
Dall'Oglio MF, Leite KR. Stage, grade and behavior of bladder urothelial carcinoma defined by the microRNA expression profile. J Urol. 2012; 188: 1951-6. doi: 10.1016/j.juro.2012.07.004.

40. Wang S, Xue S, Dai Y, Yang J, Chen Z, Fang X, Zhou W, Wu W, Li Q. Reduced expression of microRNA-100 confers unfavorable prognosis in patients with bladder cancer. Diagn Pathol. 2012; 7: 159. doi: 10.1186/1746-1596-7-159.

41. Feng B, Wang R, Chen LB. MiR-100 resensitizes docetaxelresistant human lung adenocarcinoma cells (SPC-A1) to docetaxel by targeting Plk1. Cancer Lett. 2012; 317: 18491. doi: 10.1016/j.canlet.2011.11.024.

42. Xiao F, Bai Y, Chen Z, Li Y, Luo L, Huang J, Yang J, Liao H, Guo L. Downregulation of HOXA1 gene affects small cell lung cancer cell survival and chemoresistance under the regulation of miR-100. Eur J Cancer. 2014; 50: 1541-54. doi: 10.1016/j.ejca.2014.01.024.

43. Zhang Y, Shi ZL, Yang X, Yin ZF. Targeting of circulating hepatocellular carcinoma cells to prevent postoperative recurrence and metastasis. World J Gastroenterol. 2014; 20: 142-7. doi: 10.3748/wjg.v20.i1.142.

44. Chen P, Zhao X, Ma L. Downregulation ofmicroRNA-100 correlates with tumor progression and poor prognosis in hepatocellular carcinoma. Mol Cell Biochem. 2013; 383: 49-58.

45. Tovar V, Alsinet C, Villanueva A, Hoshida Y, Chiang DY, Sole M, Thung S, Moyano S, Toffanin S, Minguez B, Cabellos L, Peix J, Schwartz M, et al. IGF activation in a molecular subclass of hepatocellular carcinoma and preclinical efficacy of IGF-1R blockage. J Hepatol. 2010; 52: 550-9. doi: 10.1016/j.jhep.2010.01.015.

46. Zhang H, Luo XQ, Zhang P, Huang LB, Zheng YS, Wu J, Zhou H, Qu LH, Xu L, Chen YQ. MicroRNA patterns associated with clinical prognostic parameters and CNS relapse prediction in pediatric acute leukemia. PLoS One. 2009; 4: e7826. doi: 10.1371/journal.pone.0007826.

47. Chen J, Zheng B, Wang C, Chen Y, Du C, Zhao G, Zhou Y, Shi Y. Prognostic role of microRNA-100 in various carcinomas: evidence from six studies. Tumour Biol. 2014; 35: 3067-71. doi: 10.1007/s13277-013-1398-3.

48. Zeng X, Zhang Y, Kwong JS, Zhang C, Li S, Sun F, Niu $\mathrm{Y}, \mathrm{Du}$ L. The methodological quality assessment tools for preclinical and clinical studies, systematic review and meta-analysis, and clinical practice guideline: a systematic review. J Evid Based Med. 2015; 8: 2-10. doi: 10.1111/ jebm.12141.

49. Zamora J, Abraira V, Muriel A, Khan K, Coomarasamy A. Meta-DiSc: a software for meta-analysis of test accuracy data. BMC Med Res Methodol. 2006; 6: 31. doi: 10.1186/1471-2288-6-31.

50. Higgins JP, Thompson SG, Deeks JJ, Altman DG. Measuring inconsistency in meta-analyses. BMJ. 2003; 327 : 557-60. doi: 10.1136/bmj.327.7414.557. 